\title{
The effect of beam scan strategies on the microstructure and mechanical properties of additive manufacturing Ti-6Al-4V builds
}

\author{
Meiyue Shao ${ }^{1}$, Sriram Vijayan ${ }^{2}$ and Joerg Jinschek ${ }^{1}$ \\ ${ }^{1}$ the Ohio State University, Columbus, Ohio, United States, ${ }^{2}$ the Ohio State University, United States
}

Additive manufacturing (AM) is an attractive and widely applied manufacturing approach to produce complex functional components, due to its advantages [1-3] such as high geometric design flexibility, low 'buy-to-fly' ratio, and reduced need for expensive tooling. Ti-6Al-4V(Ti64) is one of the most widely used alloys in AM process. Due to its excellent properties including high strength to weight ratio, high fatigue life, high biocompatibility and outstanding corrosion resistance, AM Ti64 is now widely applied in the automobile, aerospace, energy and biomedical industries. Electron beam melting (EBM) is an AM process that employs a high-energy electron beam as a fine-controlled moving heat source to melt prealloyed metal powder deposited on a substrate in a 'layer-by-layer' process. The layer-by-layer addition of powder, followed by melting and solidification over the 'previously melted' layer results in steep thermal gradients $(\mathrm{G})$. And, during the entire EBM process, a single layer of material has experienced multiple thermal cycles, which leads to microstructure and property anisotropy inside the EBM build. The spatio-temporal variations of $\mathrm{G}$ and solidification rate $(\mathrm{R})$ of the melt pool, subsequently result in the typical columnar grain growth across multiple build layers in AM builds. The primary beam parameters (such as beam current, beam speed, focus offset, energy density, and here the beam scan strategy) have significant effect on the $\mathrm{G}$ and $\mathrm{R}$, giving us opportunity to control over the resulting grain structure (columnar, mixed, or equiaxed) and the subsequent mechanical properties.

Previous research reported that scan strategies with unique fill patterns could be used to control the microstructure site specifically in Ni-alloys. In case of Inconel (IN) 718, a spot scan strategy was used site-specifically, to promote transient thermal gradients and control the liquid/solid interface velocity [4]. This was later expanded by using an 'ordered' spot scan (OS) strategy to transform the microstructure from columnar to equiaxed [5, 6]. By using different EBM scan strategies, $\mathrm{G}$ and $\mathrm{R}$ values can be tuned to desired values, thereby opening up a process parameter space to design site-specific microstructure for a given geometry [6].

Studies investigating the role of different beam scan strategies to control site specific microstructure in Ti64 are still very limited [7]. Here, we compare the effect of three different EBM scan strategies, i.e. raster linear scan (LS), ordered spot scan (OS) and random spot scan(RS), building three identical $15 \times 15$ x $25 \mathrm{~mm}^{3}$ Ti64 blocks (as shown in figure1a). Site-specific microstructure characterization was conducted across the top and bottom build ( $\mathrm{x}-\mathrm{y}$ ) planes as well as along the build ( $\mathrm{x}-\mathrm{z}$ ) direction (see figure 1b\&c). Using scanning electron microscopy (SEM) and large-area electron backscatter diffraction (EBSD), variations in microstructure and crystallographic texture, such as $\alpha$ lath thickness, prior beta $\beta$ grain size and texture orientation, $\alpha / \alpha$ lath boundary (LB) distributions, are investigated with respect to the applied scan strategy (figure 2). Both spot scan strategies result in coarser $\alpha$ laths and smaller prior $\beta$ grains than Raster scan[7]. The combined fraction of type 2 and type $4 \alpha / \alpha$ LBs measured in the three samples was found to be greater than the expected combined fraction for a random distribution of $\alpha$ variants. Additional site-specific microhardness measurements (Vickers Pyramid Number (HV) were performed and correlated with the microstructural features( $\alpha$ lath thickness) from the corresponding region (see figure 1d), indicating systematically smaller HV values in our spot scan samples. 
Currently, the structure and composition of the by-EBSD-identified dominant type 2 and type $4 \alpha / \alpha$ LBs are further characterized using scanning/transmission electron microscopy, supporting our efforts to develop a deep understanding of the microstructure-property relationship in EBM Ti64.

The authors acknowledge the scientific and technical input and support from ORNL's Manufacturing Demonstration Facility (MDF), and especially acknowledge the contributions of Sabina Kumar (University of Tennessee Knoxville, UTK), and Dr. Suresh Babu (ORNL/UTK) for several fruitful discussions. Further, the authors wish to thank Dr. Adam Pilchak (AFRL) for providing us the BOR code and Brian Welk (OSU) for his valuable inputs and discussion. Electron microscopy was performed at the Center for Electron Microscopy and Analysis (CEMAS) at The Ohio State University. This project is supported by the Australia-US Multidisciplinary University Research Initiative (MURI) program under ONR award number N00014-18-1-2794, project "Rationalization of Liquid/Solid and Solid/Solid Interphase Instabilities During Thermal-Mechanical Transients of Metal Additive Manufacturing". Any opinions, findings, and conclusions or recommendations expressed in this material are those of the author(s) and do not necessarily reflect the views of the Office of Naval Research.

(a)

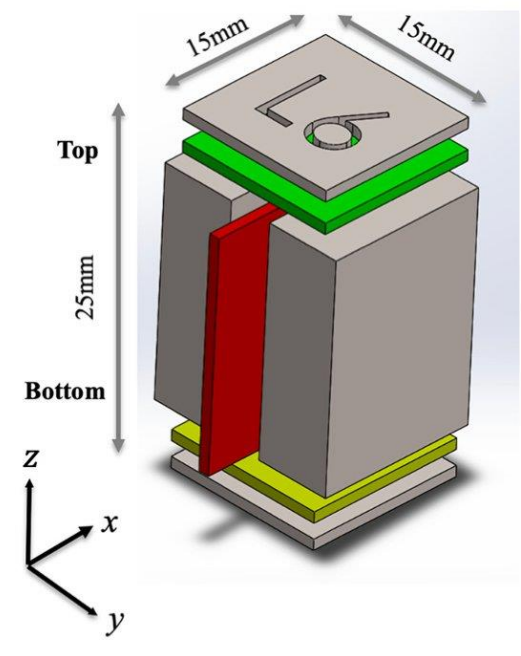

(b) SEM scan areas

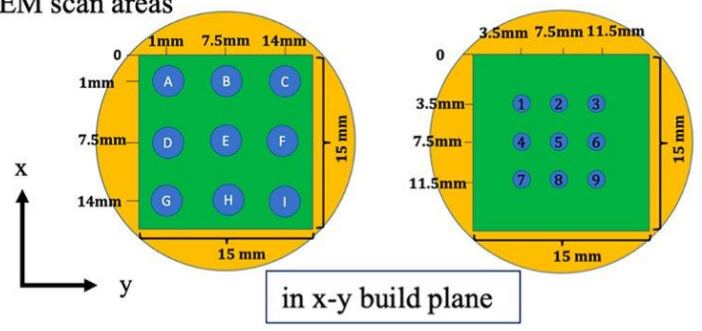

(c) EBSD scan areas

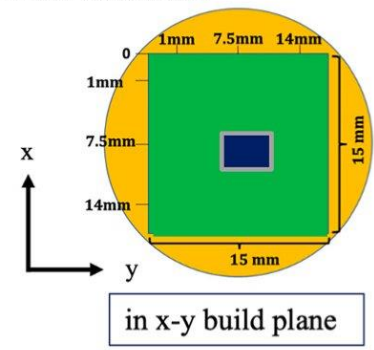

(d) Microhardness test locations

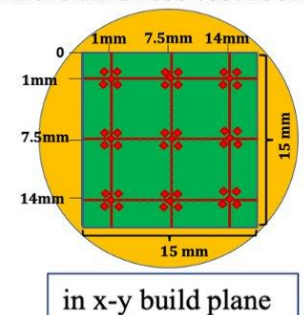

in $\mathrm{x}-\mathrm{y}$ build plane

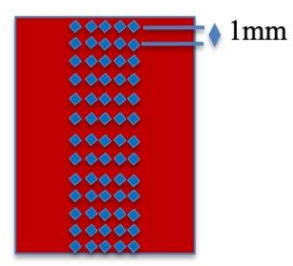

in build z-direction

Figure 1. Figure 1(a) Schematic of a Ti64 AM test build, indicating dimensions of the block $(15 \mathrm{~mm} \times$ $15 \mathrm{~mm} \times 25 \mathrm{~mm}$ ) and locations for SEM and EBSD analysis at the bottom (yellow) and top(green) in the build plane (x-y plane) and in the build direction (red, $x-z$ plane); (b) Schematics indicating the 18 locations in each $x-y$ plane to acquire high-contrast SEM backscattered electron (BSE) images - locations A-I (in order to identify general trends in the build plane) and locations 1-9 (in order to identify trends at the center of the build);(c) Schematics indicating the locations for large area EBSD scans: $800 \mu \mathrm{m} \times 650$ $\mu \mathrm{m}$ in $\mathrm{x}-\mathrm{y}$ build plane and $2000 \mu \mathrm{m} \times 1600 \mu \mathrm{m}$ in $\mathrm{x}-\mathrm{z}$ build direction; (d) Schematics indication the locations for Vickers hardness test in $\mathrm{x}-\mathrm{y}$ build plane(same as the SEM locations A-I) and in $\mathrm{x}-\mathrm{z}$ build direction 

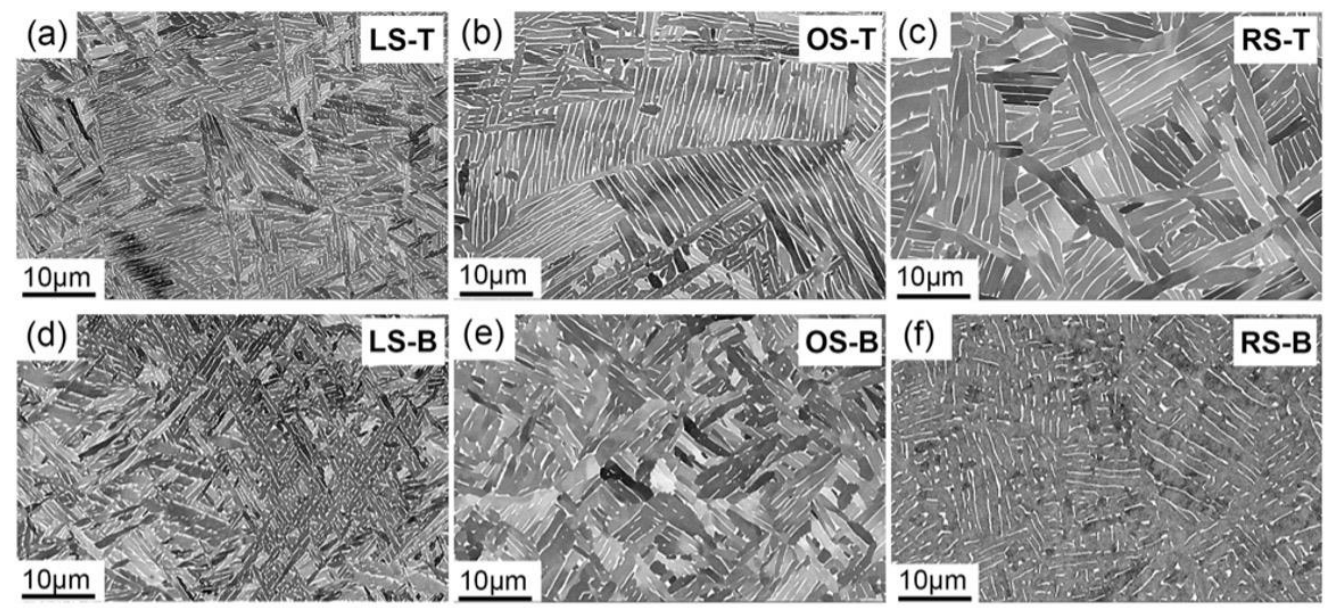

Figure 2. Figure 2 Backscattered electrons (BSE)- SEM images, taken at center of $x-y$ build planes from top section of (a) LS sample (LS-T), (b)OS sample (OS-T), and (c) RS sample (RS-T) as well as from bottom section of (d) LS sample (LS-B), (e) OS sample (OS-B), and (f) RS sample (RS-B), respectively. (adapted from Ref.7)

\section{References}

[1] W.E. Frazier, Metal additive manufacturing: a review, J. Mater. Eng. Perform. 23(2014) 1917-1928

[2] E. Herderick, Additive manufacturing of metals: A review, Mater. Sci. Technol. Conf. Exhib. 2011, MS T'11 2 (2011) 1413-1425.

[3] C. Körner, Additive manufacturing of metallic components by selective electron beam melting - a review, Int. Mater. Rev. 61 (2016) 361-377

[4] R.R. Dehoff, M. Kirka, W.J. Sames, H. Bilheux, A.S. Tremsin, L.E. Lowe, S.S. Babu, Site specific control of crystallographic grain orientation through electron beam additive manufacturing, Mater. Sci. Technol. (United Kingdom). 31 (2015) 931-938

[5] M.M. Kirka, Y. Lee, D.A. Greeley, A. Okello, M.J. Goin, M.T. Pearce, R.R. Dehoff, Strategy for texture Management in Metals Additive Manufacturing, Jom. 69 (2017) 523-531

[6] Y.S. Lee, M.M. Kirka, R.B. Dinwiddie, N. Raghavan, J. Turner, R.R. Dehoff, S.S. Babu, Role of scan strategies on thermal gradient and solidification rate in electron beam powder bed fusion, Addit. Manuf. 22 (2018) 516-527

[7] M. Shao, S. Vijayan, P. Nandwana, and J.R. Jinschek. The effect of beam scan strategies on microstructural variations in Ti-6Al-4V fabricated by electron beam powder bed fusion, Materials \& Design. 196 (2020) 109165. 